\title{
On the use of IMAGE FUV for estimating the latitude of the open/closed magnetic field line boundary in the ionosphere
}

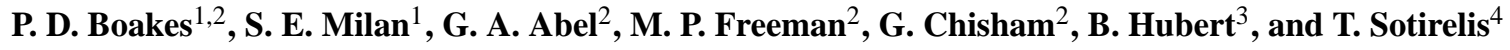 \\ ${ }^{1}$ Department of Physics and Astronomy, University of Leicester, Leicester, LE1 7RH, UK \\ ${ }^{2}$ British Antarctic Survey, Natural Environment Council, Cambridge, UK \\ ${ }^{3}$ Laboratory of Planetary and Atmospheric Physics, University of Liege, Belgium \\ ${ }^{4}$ Applied Physics Laboratory, Johns Hopkins University, Laurel, MD, USA
}

Received: 9 May 2008 - Accepted: 29 July 2008 - Published: 16 September 2008

\begin{abstract}
A statistical comparison of the latitude of the open/closed magnetic field line boundary (OCB) as estimated from the three far ultraviolet (FUV) detectors onboard the IMAGE spacecraft (the Wideband Imaging camera, WIC, and the Spectrographic Imagers, SI-12 and SI-13) has been carried out over all magnetic local times. A total of over 400000 OCB estimations were compared from December 2000 and January and December of 2001-2002. The modal latitude difference between the FUV OCB proxies from the three detectors is small, $<1^{\circ}$, except in the predawn and evening sectors, where the SI-12 OCB proxy is found to be displaced from both the SI-13 and WIC OCB proxies by up to $2^{\circ}$ poleward in the predawn sector and by up to $2^{\circ}$ equatorward in the evening sector. Comparing the IMAGE FUV OCB proxies with that determined from particle precipitation measurements by the Defense Meteorological Satellites Program (DMSP) also shows systematic differences. The SI-12 OCB proxy is found to be at higher latitude in the predawn sector, in better agreement with the DMSP OCB proxy. The WIC and SI-13 OCB proxies are found to be in better agreement with the DMSP OCB proxy at most other magnetic local times. These systematic offsets may be used to correct FUV OCB proxies to give a more accurate estimate of the OCB latitude.
\end{abstract}

Keywords. Ionosphere (Active experiments) - Magnetospheric physics (Auroral phenomena; Instruments and techniques)

\section{Introduction}

New insights into the physics of magnetic reconnection, including its continuity, extent and controlling factors, have been provided by remote measurements of the global and lo-

Correspondence to: P. D. Boakes

(p.boakes@ion.le.ac.uk) cal magnetic reconnection rate in the magnetospheric system (Chisham et al., 2008, and references therein). Such remote sensing requires the accurate determination of the boundary in the ionosphere between magnetic field lines of open and closed topology.

Many different ionospheric measurements may be employed to determine the location of the open/closed magnetic field line boundary (OCB) in the ionosphere. These include optical measurements from all-sky cameras, imaging spacecraft and meridian-scanning photometers (Blanchard et al., 1995, 1997; Sandholt et al., 1998; Brittnacher et al., 1999), particle precipitation signatures from low-altitude spacecraft (Newell et al., 1991, 1996; Sotirelis and Newell, 2000) and measurements from incoherent and coherent scatter radars (Baker et al., 1995; Blanchard et al., 1996, 2001; Milan et al., 1999; Chisham et al., 2001, 2002; Lester et al., 2001; Chisham and Freeman, 2003), or a combination of all of these (Milan et al., 2003). Unfortunately, most of these measurements suffer from only partial coverage of the auroral oval at any one time, making a global determination of the OCB difficult. Particle precipitation boundaries (PPB) measured by low-altitude spacecraft (such as the Defence Meteorological Satellite Program (DMSP) spacecraft) provide the most direct and precise determination of boundaries between different plasma regions, including the OCB. However, these spacecraft only cross the OCB in two magnetic local time (MLT) sectors in each hemisphere in any one orbit ( $\sim 100 \mathrm{~min}$ for the DMSP satellite) and therefore can not be used to determine the global location of the OCB.

Currently the best method for estimating the global location of the OCB is from images of the aurora taken by satellites. However, recent studies have shown that there are systematic differences of up to a few degrees between the latitude of the boundaries determined from auroral images and the in-situ particle precipitation measurements (Kauristie et al., 1999; Baker et al., 2000; Carbary et al., 2003). Carbary et al. (2003) studied the statistical discrepancy between the

Published by Copernicus Publications on behalf of the European Geosciences Union. 
DMSP OCB proxy and an OCB proxy derived from auroral images taken in the Lyman-Birge-Hopfield "long" (LBHL) band (164-178 $\mathrm{nm}$ ) by the Ultra-Violet Imager (UVI) on the Polar spacecraft. They showed that the largest discrepancy occurs in the predawn sector ionosphere, with the DMSP proxy found up to $\sim 3.5^{\circ}$ poleward of the UVI proxies at 05:00 MLT. A very similar discrepancy was found in the predawn sector ionosphere by Chisham et al. (2005), comparing the DMSP OCB proxy with an OCB proxy provided by the Super Dual Auroral Radar Network (SuperDARN) Spectral Width Boundary (SWB). Wild et al. (2004) presented a case study in which the latitudes of both the SWB OCB proxy and the OCB estimated using the Wideband Imaging Camera (WIC) (140-160 nm) onboard the IMAGE spacecraft were placed several degrees equatorward of the OCB PPB proxies determined from Cluster, DMSP and FAST spacecraft measurements obtained simultaneously in the predawn sector. However they showed that the OCB determined from the Spectrographic Imager SI-13 ( 133$138 \mathrm{~nm})$ on board IMAGE was closely co-located with the OCB PPB proxies. They concluded that in the dawn sector ionosphere the SWB and the poleward boundary of auroral emissions measured by WIC were unreliable proxies for the OCB in this case, with the poleward boundary of SI-13 emissions providing a more accurate proxy. Other studies have used the poleward boundary of SI-12 auroral emission $(\sim 119-124 \mathrm{~nm})$ in preference to WIC or SI-13 OCB proxies due to the reduced level of dayglow, making boundary estimation possible even in summer months (Hubert et al., 2006). However, these studies did not consider if SI-12 gave the most reliable estimation of the OCB location. Using IMAGE FUV data, Mende et al. (2003) also noted that the proton aurora was found to be displaced equatorward of the electron aurora for dusk local times and poleward for dawn local times during all phases of substorms.

In this paper we compare, statistically, the magnetic latitude of the OCB as estimated from auroral images from the WIC, SI-12 and SI-13 Far Ultra-Violet (FUV) detectors onboard the IMAGE satellite (FUV OCB proxies) and from DMSP particle precipitation measurements (DMSP OCB proxy). In Sect. 2 we set out the method of FUV OCB estimation using a modified method of Carbary et al. (2003) and compare the FUV OCB proxies. In Sect. 3 we compare the FUV OCB proxies with the DMSP OCB proxy. We discuss these results and their implications for accurately estimating the latitude of the OCB at all magnetic local times in Sect. 4, and finally we summarise our findings in Sect. 5.

\section{Comparison between IMAGE FUV OCB proxies}

The FUV imager onboard the IMAGE satellite (Mende et al., 2000a, b) consists of three detectors observing the aurora with a temporal resolution of two minutes (limited by the spin period of the spacecraft). The Wideband Imag- ing Camera (WIC) has a bandwidth of 140-180 nm, observing emissions from the N2 Lyman-Birge-Hopfield band and atomic NI lines. The Spectrographic Imager SI-12 observes the proton aurora with a pass band of $5 \mathrm{~nm}$ centered on the $121.82 \mathrm{~nm}$ Doppler-shifted Lyman- $\alpha$ and the SI-13 detector observes the "excited electron aurora" with a pass band of $5 \mathrm{~nm}$ centered on the $135.6 \mathrm{~nm}$ OI doublet. WIC offers the best spatial resolution of approximately $50 \mathrm{~km}$ at apogee, compared to approximately $100 \mathrm{~km}$ offered by both spectrographic imagers. IMAGE was operational from May 2000 to December 2005, when the spacecraft failed. In this study we use data from December 2000 and January and December of 2001 and 2002. After this date the camera pointing became less reliable such that the position of the auroral oval may be offset from its true position by up to $1^{\circ}$ of latitude. We only use images from December and January as dayglow is much less prominent in these months; whilst in other months dayglow often swamps the dayside auroral oval, particularly in the WIC data, making the estimation of the dayside OCB latitude impossible.

\subsection{Identification of IMAGE FUV OCB proxies}

Intensity-latitude profiles of the auroral oval are created by averaging the FUV intensities in bins of one degree of magnetic latitude and one hour of MLT. Examples of intensitylatitude profiles are shown in Fig. 1a-c, where the binned data are represented by diamonds. All data presented in this paper are in AACGM coordinates.

We use a modification of the method of Carbary et al. (2003) (hereafter called the FUV OCB estimation method) to estimate the location of the OCB in each $1 \mathrm{~h}$ MLT sector. This method involves fitting a Gaussian function plus a quadratic background to the intensity-latitude profiles from a single $1 \mathrm{~h}$ MLT sector. Although the method of Carbary et al. (2003) was developed using data from the Polar Ultraviolet Imager, Lam et al. (2006) have shown that the same method can be successfully applied to other auroral imagers.

The solid curves in Fig. 1a-c represent a fit to the binned data using a non-linear least-square fit (using the IDL Gaussfit routine) to the following function:

$$
T(\lambda)=A_{0} \exp \left[-\frac{1}{2}\left(\frac{\lambda-A_{1}}{A_{2}}\right)^{2}\right]+A_{3}+A_{4} \lambda+A_{5} \lambda^{2}
$$

where $\lambda$ is the magnetic latitude and $A_{0}-A_{5}$ are coefficients of the fit. The Gaussian part of Eq. (1) is assumed to represent the auroral oval and the quadratic background is assumed to include extraneous airglow, off-axis radiance, or unwanted non-oval aurora. A fit is assumed to be successful if the following criteria are satisfied:

1. The intensity of the Gaussian, $A_{0}$, exceeds 5 counts (we use corrected counts from the IMAGE data, where corrections for flatfield, gain of the MCP etc. have been 

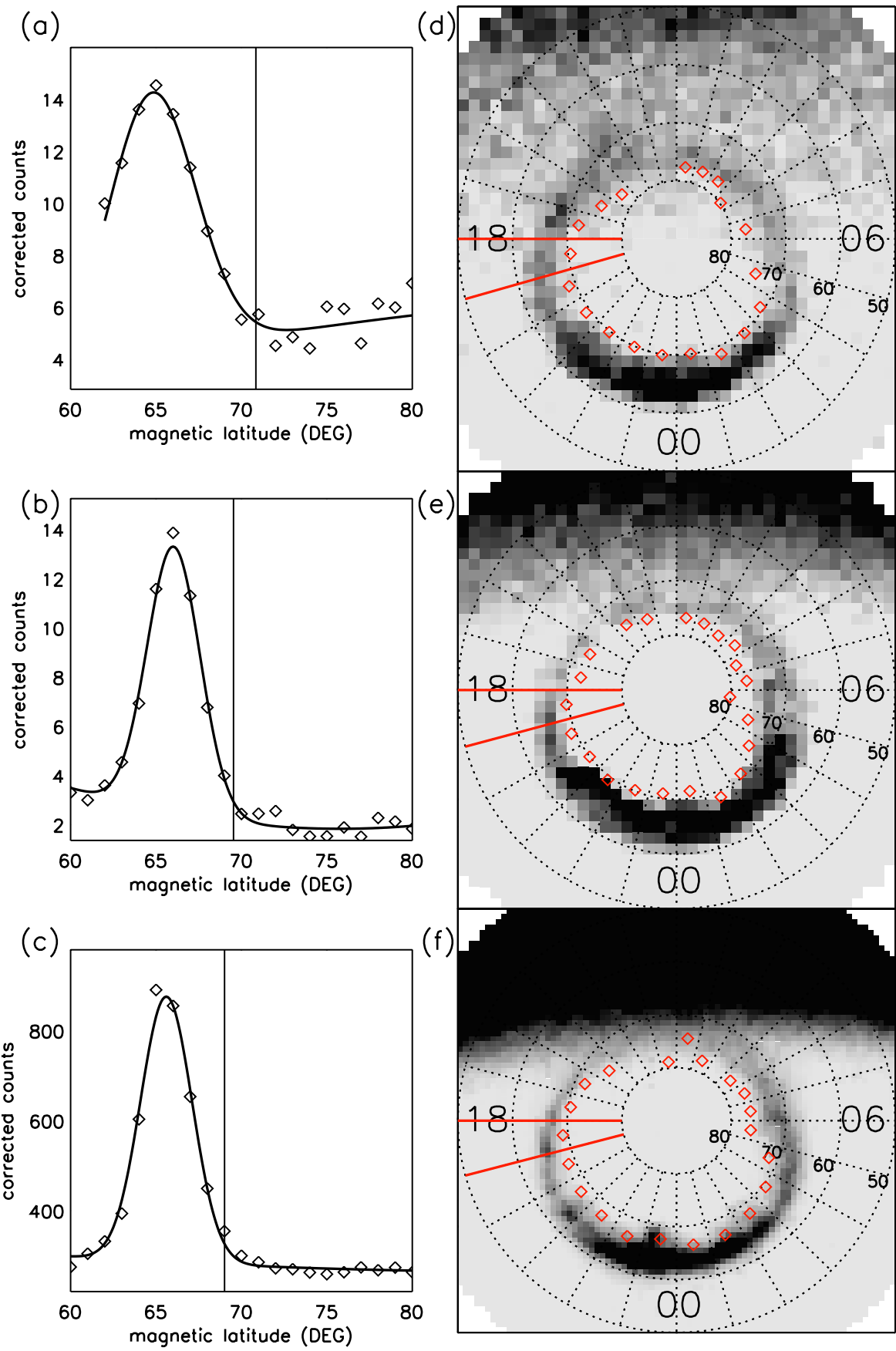

Fig. 1. (a-c) Intensity-latitude profiles (left-hand column) across a $1 \mathrm{~h}$ MLT sector (18:00-19:00 MLT) and (d-f) images of the auroral oval (right-hand column), with magnetic local noon at the top of the figure, for 20:40:09 on 26 December 2000. Rows correspond to data from three different IMAGE FUV detectors, SI-12 (top), SI-13 (middle) and WIC (bottom). Average intensities in bins of one degree magnetic latitude are shown by diamonds and the Gaussian plus quadratic background fits to these points are shown by the solid curves. The position of the OCB proxies are marked by the vertical solid lines and overplotted on the auroral images by red diamonds.

applied so that one Rayleigh of emission always produces the same number of counts wherever in the CCD).

2. The magnetic latitude of the peak of the Gaussian, $A_{1}$, is between $50^{\circ}$ and $85^{\circ}$ magnetic latitude.
3. The Gaussian FWHM $\left(\Delta \lambda=2 A_{2} \sqrt{2 \ln 2}\right)$ exceeds $1^{\circ}$.

4. The ratio of the background at the center of the Gaussian $\left(B=A_{3}+A_{4} \cdot A_{1}+A_{5} \cdot A_{1}^{2}\right)$ to the peak of the Gaussian, $A_{0}$, does not exceed 0.2 . 
5. $\Delta \lambda<0.3\left(\lambda_{\max }-\lambda_{\min }\right)$. The Gaussian width spans less than $30 \%$ of the latitude bins which have non-zero intensity.

The latitude of the OCB, $\lambda_{\mathrm{OCB}}$ (shown by the vertical lines in Fig. 1a-c and overplotted on the auroral images (Fig. 1df) by diamonds), is assumed to be displaced poleward of the centre of the auroral oval by the Gaussian FWHM such that

$\lambda_{\mathrm{OCB}}=A_{1}+\Delta \lambda$

By visually inspecting the data we note that on occasion the FUV OCB estimation method produces a latitude profile meeting the given criteria above but which results in an apparently inaccurate boundary estimation (displaced several degrees in latitude from the location estimated by eye). We have identified two particular reasons why these errors occur and correct as follows:

1. The auroral oval can often display a double oval configuration (Elphinstone et al., 1996) causing the intensitylatitude profiles to have a multiple peaked nature with two or more Gaussian like components. Normally the Gaussian fitting procedure will fit to the most equatorward peak and include the more poleward peaks in the quadratic background part of the fit. To find the most accurate location of the OCB we must include these more poleward peaks in the Gaussian part of the fit. We do this by searching for the most poleward peak meeting the criteria $1-5$ as follows. The intensity-latitude profile must decrease in intensity over at least two degrees of magnetic latitude in the poleward direction from the latitude of the last peak found, followed by an increase over at least two degrees of latitude. Once a new maximum in intensity is found the intensity must decrease again over at least two degrees of magnetic latitude. The Gaussian fitting procedure is then applied to the most poleward peak identified below $85^{\circ}$ magnetic latitude by including only latitudes more poleward of the minimum between the most poleward peak and the previous peak in the fitting routine. This will also effectively remove dayglow from intensity-latitude profiles when a clear drop in intensity exists between the poleward edge of dayglow and the equatorward edge of the auroral oval.

2. Dayglow often extends close to or onto the auroral oval, often completely swamping it in the summer months. The poisson noise associated with the dayglow can be larger than the auroral signature itself. In most cases the criteria 1-5 reject any identification of the OCB. However occasionally this is not the case and the Gaussian function is fit to the dayglow emission rather than the auroral oval emission, placing the OCB estimate at the poleward edge of the dayglow, as seen at 11:0012:00 MLT in Fig. 1f. Extra criteria are used to try and eliminate these cases as follows. On the dayside
(06:00-18:00 MLT) we reject an OCB proxy if it has been placed over five degrees poleward or equatorward of the proxy found in the previous MLT sector. If the previous MLT sector has not produced a proxy we use the next earlier MLT. If this has also not produced a proxy the MLT sector tested is rejected.

If an OCB proxy has not been identified by this point, in a bid to remove dayglow from the fit, we shift the lower limit of the latitudes included when creating the intensity-latitude profiles to higher latitudes in steps of one degree until an estimation of the OCB can be found (by reapplying all the above FUV OCB estimation method) up to a maximum latitude lower limit of $80^{\circ}$. A similar correction as above is applied on the nightside, 00:00-06:00 MLT and 18:00-24:00 MLT, if the OCB is located over $8^{\circ}$ poleward or equatorward of adjacent MLTs. The larger value on the nightside is used as we find (from visual inspection) that the latitude of the OCB can legitimately change by between 5-8 degrees, particularly around the auroral bulge where at substorm onset the latitude of the OCB is rapidly changing.

These extra criteria remove many erroneous OCB estimations. However, we can not catch all bad estimations, as seen in Fig. 1f 11:00-12:00 MLT, where the OCB proxy is placed equatorward of the poleward edge of the WIC auroral emission but is just within the five degree condition. These criteria will also not catch poorly estimated OCB proxies if several poorly estimated proxies have been made in several adjacent MLT bins.

\subsection{Comparison between FUV detectors}

We apply the FUV OCB estimation method to every FUV auroral image obtained by IMAGE from the months of December 2000 and December and January 2001-2002. Comparing FUV OCB proxies from different cameras at the same UT and MLT gives a total of 499273 comparisons between WIC and SI-12 OCB proxies, 543505 comparisons between WIC and SI-13 OCB proxies and 408753 comparisons between SI-12 and SI-13 OCB proxies, and over 4000 comparisons in each hour MLT bin.

Let us define the latitude of WIC, SI-12 and SI-13 OCB proxies as $\lambda_{\text {WIC }}, \lambda_{\text {SI-12 }}$ and $\lambda_{\text {SI-13 }}$, respectively. Figure 2 shows distributions of the latitude differences between matched WIC and SI-12 OCB proxies $\left(\lambda_{\text {WIC }}-\lambda_{\mathrm{SI}}-12\right)$ for four 1-h MLT bins. Positive values of latitude difference are defined such that the WIC OCB proxy is at higher latitudes than the SI-12 OCB proxy. Crosses in Fig. 2 represent the number of occurrences of latitude differences between WIC and SI-12 OCB proxies in bins of $0.2^{\circ}$ magnetic latitude. In order to reduce small statistical uncertainty smoothing has been applied to the distributions three times using boxcar averaging over five points. Large boundary differences can be seen in the tails of these distributions. Visual inspection of these points shows that they occur when a poor boundary estimation has been made, and has not been caught by the criteria 

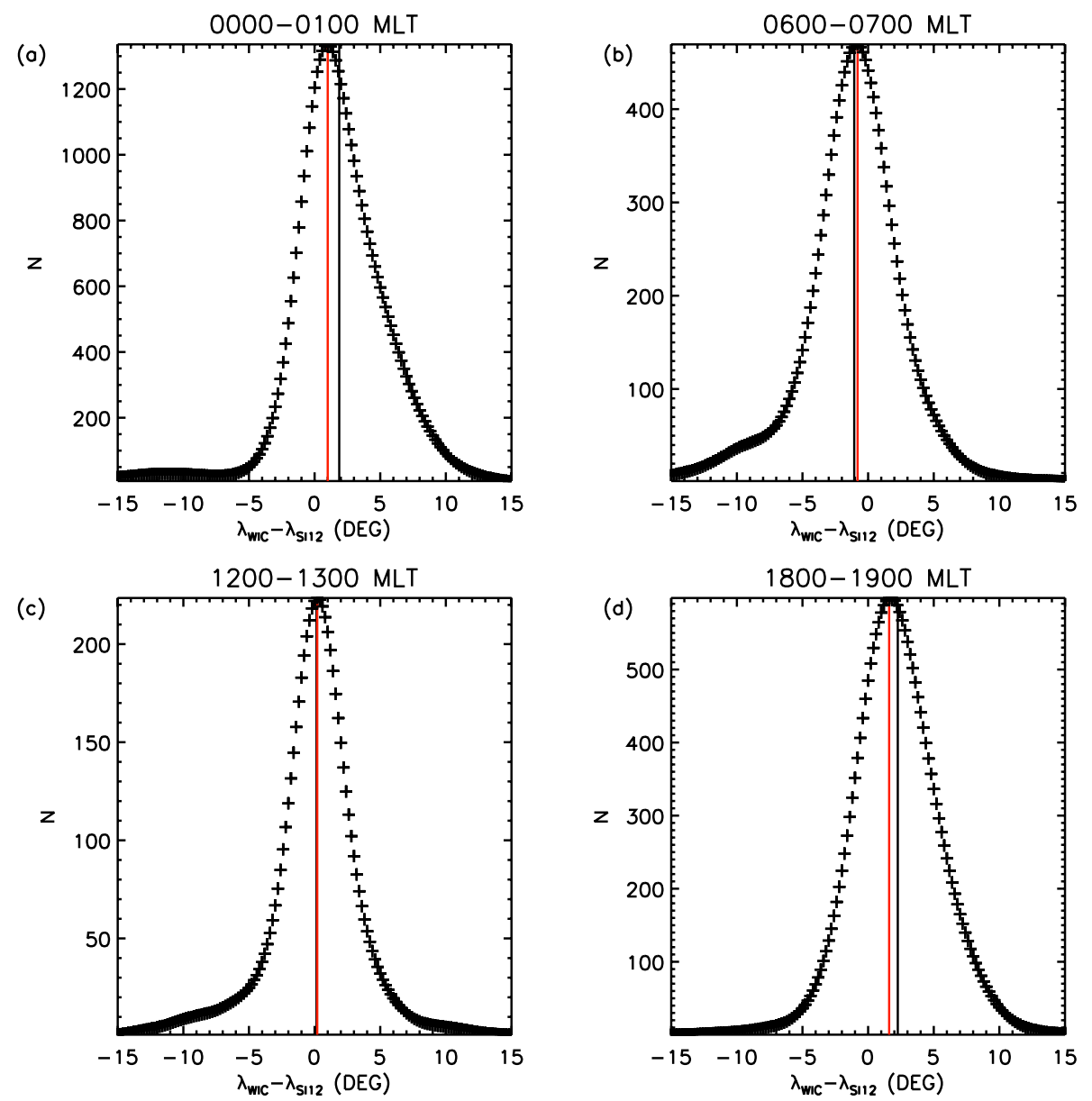

Fig. 2. Frequency distribution of the difference between the latitude of WIC and SI-12 OCB proxies for 00:00-01:00, 06:00-07:00, 12:0013:00 and 18:00-19:00 MLT. Crosses represent the number of boundary differences in $0.2^{\circ}$ bins of magnetic latitude, red vertical lines represent the mode of the distribution and black vertical lines the mean.

discussed above, in one or both of the FUV cameras. We characterise the latitude difference between the FUV OCB proxies by the mode of the distribution (shown by the red vertical lines in Fig. 2), since this is insensitive to the presence of these outliers. Black vertical lines represent the mean of the distributions.

Figure 3 shows the variation of the modal FUV OCB proxy latitude differences with MLT, for $\lambda_{\text {WIC }} \lambda_{\text {SI-12 }}$ (blue), $\lambda_{\text {WIC }}{ }^{-}$ $\lambda_{\mathrm{SI}-13}$ (black) and $\lambda_{\mathrm{SI}-13}-\lambda_{\mathrm{SI}-12}$ (yellow). To put the variation in context we also provide some information on the width of the distributions and the error on the mode. The width of the latitude difference distributions (Fig. 2) is estimated by moving out from the mode (red line Fig. 2) in steps of one 0.2 degree bin in both positive and negative directions to find the latitude range within which $68 \%$ of the points lie (which would be equal to the standard deviation for a Gaussian). We estimate the error on the mode by dividing the width by $\sqrt{N}$, where $N$ is the number of points in each bin of MLT (equivalent to the standard error on the mean for a Gaussian). The estimated widths are approximately $2^{\circ}$ and the estimated errors on the mode are $<0.01^{\circ}$.

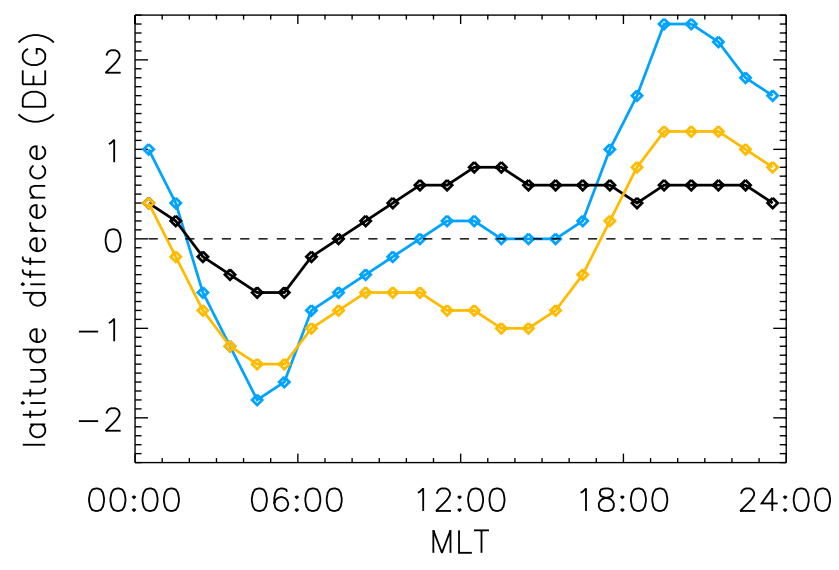

Fig. 3. Mode of OCB proxy latitude differences for $\lambda$ WIC $-\lambda$ SI -12 (blue), $\lambda_{\text {WIC }}-\lambda_{\text {SI }-13}$ (black) and $\lambda_{\text {SI }-13}-\lambda_{\text {SI-12 }}$ (yellow). The estimated widths of the distributions (explained in the text) are of order $2^{\circ}$ and the estimated errors on the modes are $<0.01^{\circ}$. 
Table 1. Coefficients of the weighted second-order fit to modal latitude differences between DMSP OCB proxies and Polar UVI proxies (from Carbary et al., 2003) and IMAGE FUV OCB proxies. $N$ is the number of OCB proxies compared in each comparison.

\begin{tabular}{ccccc}
\hline & Carbary et al. (2003) & WIC & SI-12 & SI-13 \\
\hline$C_{0}$ & 0.80 & -0.12 & 0.39 & 0.57 \\
$C_{1}$ & 1.11 & 1.13 & 1.27 & 0.99 \\
$D_{1}$ & 1.48 & 1.08 & -0.17 & 0.80 \\
$C_{2}$ & -0.79 & -0.12 & -0.46 & -0.07 \\
$D_{2}$ & 0.38 & 1.06 & -0.03 & 1.02 \\
$N$ & 11244 & 2462 & 1704 & 1838 \\
\hline
\end{tabular}

The modal latitude differences between WIC and SI-13 OCB proxies (black curve Fig. 3 ) are relatively small, $<0.8^{\circ}$. Modal latitudinal differences between WIC and SI-12 OCB proxies and between SI-13 and SI-12 OCB proxies (blue and yellow curves respectively) are also small in the 07:0017:00 MLT range. In the predawn sector SI-12 boundaries are found to be located poleward of both WIC and SI-13 by between $1^{\circ}-2^{\circ}$. Conversely SI- 12 boundaries are also found to be located at greater than 1 degree equatorward of both WIC and SI-13 in the 18:00-23:00 MLT range.

\section{Comparison with DMSP auroral boundaries}

In this section we compare the latitude of the FUV OCB proxies with the latitude ( $\left.\lambda_{\text {DMSP }}\right)$ of the OCB proxy obtained from the DMSP satellite (determined using the method of Sotirelis and Newell, 2000). We use five DMSP spacecraft (F11-15) for the years 2000-2001. On the nightside, the OCB is given by the b6 boundary which marks the transition from the poleward edge of the sub-visual drizzle region to particle fluxes below detectable levels or the first encounter with polar rain. On the dayside the OCB is given by the doc boundary, located where there is an unambiguous transition between precipitation characteristic of open and closed field lines. The central plasma sheet, boundary plasma sheet and Low Latitude Boundary Layer (LLBL) are considered to be closed, and cusp, mantle, open LLBL, polar rain and void are considered to be open. If any of these transitions are not clear, because of ambiguities in the region, then an OCB proxy is not identified (Chisham et al., 2005).

The DMSP OCB proxy is compared to the closest (in both UT and MLT) FUV OCB proxy within $\pm 0.5 \mathrm{~h}$ of MLT and \pm 10 min of UT, producing over 1700 total comparisons for each FUV detector. However, due to the orbit of the DMSP spacecraft, no DMSP boundaries are available in the Northern Hemisphere between 22:00 and 05:00 MLT.

We bin the latitude differences into bins of $0.5^{\circ}$. To reduce the statistical uncertainty and produce a smooth distribution with one clear peak the distributions have been smoothed three times using a boxcar average over five points. Figure 4 shows example distributions of latitude differences between DMSP and WIC OCB proxies for four 1-h MLT bins. The smoothed latitude differences are shown by the crosses and vertical lines show the mean (black) and modes (red) of the distributions. The distributions are skewed to the right such that there is a higher probability of finding a DMSP OCB proxy at a significantly higher latitude than the equivalent FUV proxy than there is of finding an FUV OCB proxy at a higher latitude. We suggest three possible factors which may contribute to these large differences:

1. The method of FUV OCB proxy estimation. When a poor FUV OCB proxy estimation is made (and are not caught by the criteria discussed in Sect. 2.1) it is nearly always placed equatorward of the poleward edge of the auroral oval emission and is therefore more equatorward of the equivalent DMSP boundary than should be the case.

2. The use of finite MLT and UT windows in the comparison procedure. The fine scale structure of the OCB may change by small amounts over these window sizes. Reducing the size of the windows reduces the width of the distributions but also reduces the number of comparisons available.

3. Failure of the DMSP OCB estimation procedure. The DMSP OCB estimation is made using an automatic algorithm which will occasionally fail.

The mean of the distribution, shown by the black vertical lines, is shifted towards this tail, and so we therefore use the mode of the distributions, shown by the red vertical lines, to characterise the centre of the distribution.

Figure $5 \mathrm{a}-\mathrm{c}$ shows the variation with MLT of the modal latitude differences between the FUV and DMSP OCB proxies. The horizontal lines at the center of the estimated error bars represent the mode of the latitude differences in bins of $1 \mathrm{~h}$ MLT. Thin error bars represent the estimated width and the thick error bars represent the estimated error on the mode as explained in Sect. 2.3. Due to the relatively low number of DMSP-FUV OCB proxy comparisons the estimated widths are fairly large $\left(3^{\circ}-4^{\circ}\right)$, exceeding the size of the offsets themselves, although the estimated error on the mode is smaller $\left(<1^{\circ}\right)$.

Following the method set out by Carbary et al. (2003) we fit a continuous function to the data which extrapolates into MLTs where no DMSP boundaries are available. A weighted second-order harmonic fit is applied to the modal latitude differences using a least squares fit,

$$
L(\varphi)=C_{0}+C_{1} \cos \varphi+D_{1} \sin \varphi+C_{2} \cos 2 \varphi+D_{2} \sin 2 \varphi
$$

where $\varphi$ is the angle associated with the MLT $\left(\equiv \operatorname{MLT} \times 15^{\circ}\right)$, and $C$ and $D$ are coefficients of the fit. Weights are $n / \max (n)$ where $n$ is the number of boundary comparisons in each one 
(a)

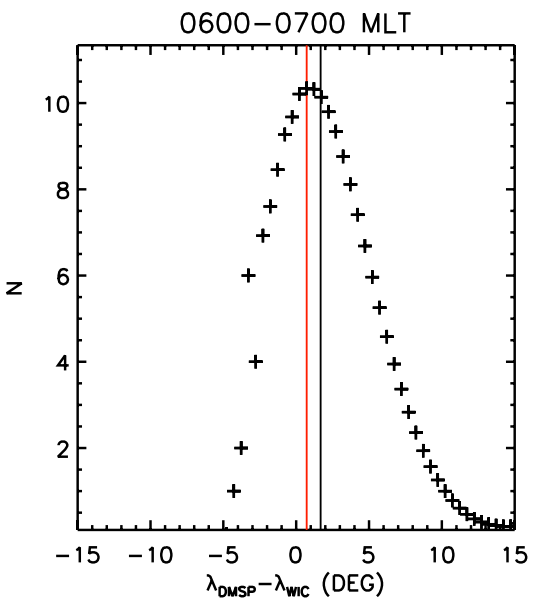

(c)

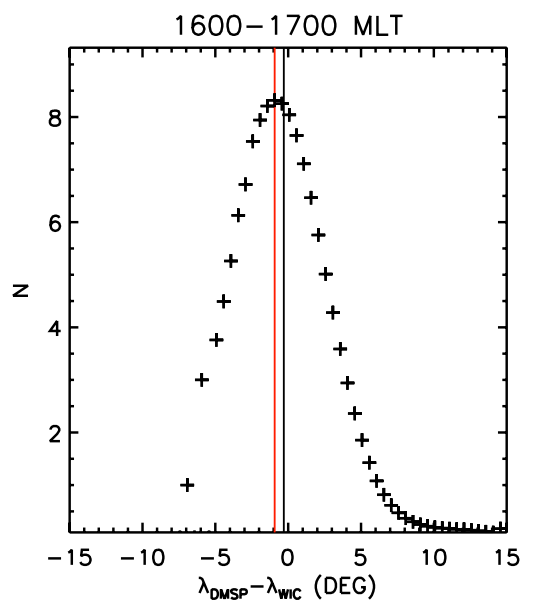

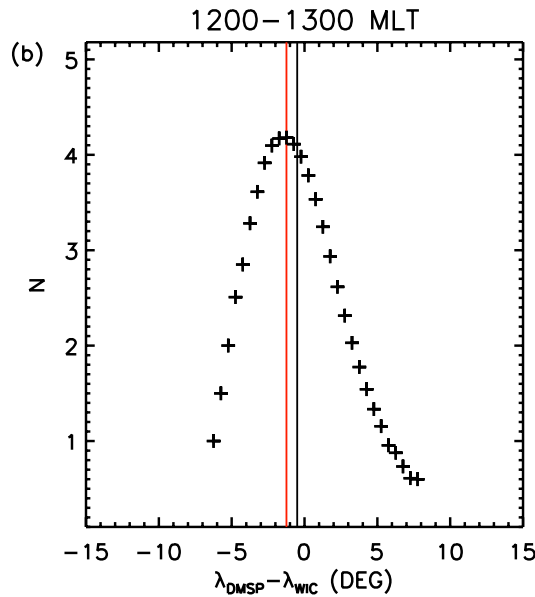

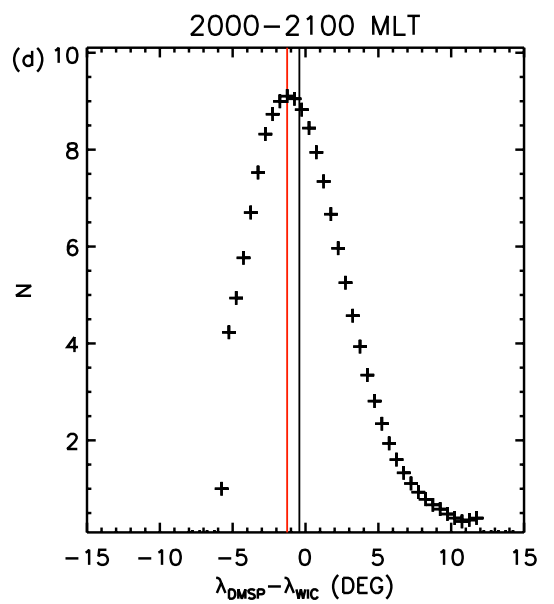

Fig. 4. Smoothed frequency distributions for $\lambda_{\text {DMSP }}-\lambda$ WIC for (a) 06:00-07:00 MLT, (b) 12:00-13:00 MLT, (c) 16:00-17:00 MLT and (d) 20:00-21:00 MLT in bins of $0.5^{\circ}$ magnetic latitude. Vertical black lines show the mean of the distributions and red lines represent the mode.

hour MLT bin. The harmonic coefficients determined by Carbary et al. (2003) are used as initial starting values for the fitting routine and are listed with the final coefficients in Table 1 along with the number of OCB proxies compared for each detector $(N)$. Figure $5 \mathrm{~d}$ shows the resultant fits and the fit of Carbary et al. (2003) for the case of DMSP and Polar UVI OCB proxies (dashed blue line).

Latitude differences between DMSP and WIC or SI-13 OCB proxies display systematic deviations grossly consistent with the previous DMSP-UVI study (Carbary et al., 2003), with the largest deviations occurring in the predawn sector. Between 07:00 and 23:00 MLT the fits to latitude differences typically differ by less than a degree. In contrast the average latitude difference between DMSP and SI-12 is larger in the evening sector (16:00-24:00 MLT) and smaller in the morning sector (00:00-07:00 MLT), by up to $2^{\circ}$.

One of the main aims of this study is to produce an estimate of any systematic offsets between the true OCB and the OCB proxies from the FUV detectors. Assuming the DMSP OCB proxy to be the best estimate of the OCB, the compar- ison between the FUV OCB proxies and DMSP OCB proxy would give us a good estimate of this. However, we note that at individual MLTs there are inconsistencies between the DMSP-FUV comparisons and the inter-FUV comparisons. Given the considerably larger number of comparisons used in the inter-FUV comparisons we would like to use these results to enhance our best estimate of the systematic offsets of the FUV OCB proxies from the (unknown) true location of the OCB. We do this by considering characteristic latitudinal offsets of the WIC, SI-12, SI-13 and DMSP proxies, which we designate as $W, P, E$, and $D$ respectively.

We relate the characteristic latitudinal offsets $W, P, E$, and $D$ at each MLT to the values shown in Figs. 3 and 5 as follows;

$$
\begin{aligned}
& W-P=\left\langle\lambda_{\mathrm{WIC}}-\lambda_{\mathrm{SI} 12}\right\rangle+a=x_{1}+a \\
& W-E=\left\langle\lambda_{\mathrm{WIC}}-\lambda_{\mathrm{SI} 13}\right\rangle+b=x_{2}+b \\
& P-E=\left\langle\lambda_{\mathrm{SI} 12}-\lambda_{\mathrm{SI} 13}\right\rangle+c=x_{3}+c \\
& D-W=\left\langle\lambda_{\mathrm{DMSP}}-\lambda_{\mathrm{WIC}}\right\rangle+d=x_{4}+d
\end{aligned}
$$



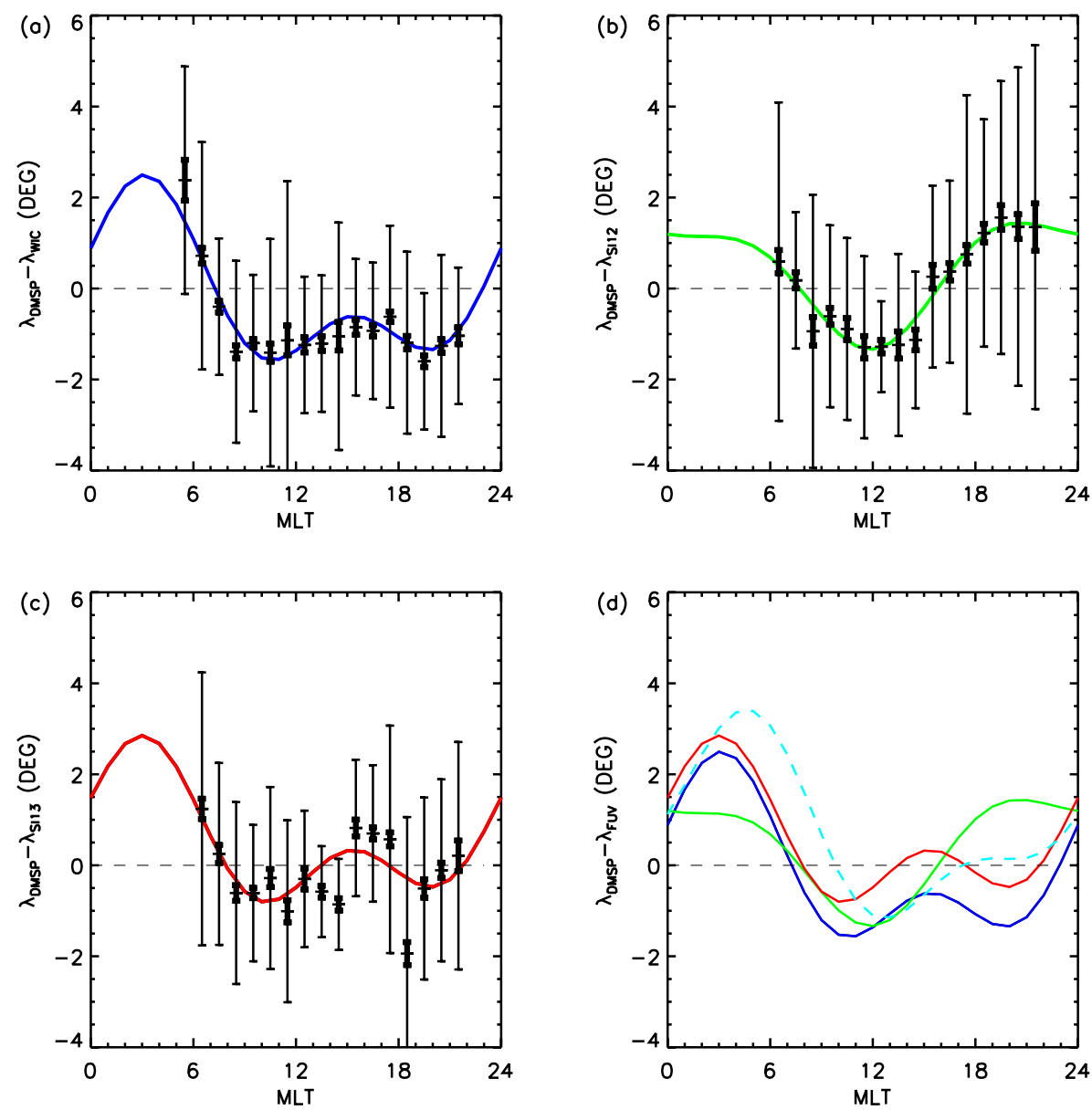

Fig. 5. Modes of OCB proxy latitude differences between FUV and DMSP, (a) $\lambda_{\text {DMSP }}-\lambda_{\text {WIC }}$, (b) $\lambda_{\text {DMSP }}-\lambda_{\text {SI }}-12$ and (c) $\lambda_{\text {DMSP }}-\lambda_{\text {SI }}-13$. Bold error bars represent the estimated error on the mode and thin error bars represent the estimated widths (see text for details). Second order harmonic fits to the OCB proxy latitude differences are shown by solid curves and compared to the fit of Carbary et al. (2003) (dashed light blue line) in panel (d).

$D-P=\left\langle\lambda_{\mathrm{DMSP}}-\lambda_{\mathrm{SI} 12}\right\rangle+e=x_{5}+e$

$D-E=\left\langle\lambda_{\mathrm{DMSP}}-\lambda_{\mathrm{SI} 13}\right\rangle+f=x_{6}+f$

where $a, b, c, d, e$, and $f$ are unknown measurement errors and $<>$ indicate an ensemble property, for which we have used the mode. By comparing the curves in Fig. 3, we find that the unknown errors, $a, b$, and $c$ are insignificant and so set them equal to zero (however, if $a, b$, and $c$ are considered to be non-zero, a full treatment leads to the same result). To optimally estimate the characteristic latitudinal offsets we minimize the sum squared error, $G=d^{2}+e^{2}+f^{2}$ by setting $d G / d D=0$, assuming $W, P$ and $E$ are known. We find

$D=\left(W+P+E+x_{4}+x_{5}+x_{6}\right) / 3$

Rearranging Eq. (10) and substituting for Eqs. (4-6) we find:

$$
\begin{aligned}
& W-D=\left(x_{1}+x_{2}-x_{4}-x_{5}-x_{6}\right) / 3 \\
& P-D=\left(-x_{1}+x_{3}-x_{4}-x_{5}-x_{6}\right) / 3
\end{aligned}
$$

$E-D=\left(-x_{2}+x_{3}-x_{4}-x_{5}-x_{6}\right) / 3$

Figure 6 presents these optimised FUV offsets relative to the DMSP proxy (Eqs. 11-13). The reference DMSP OCB proxy is shown by the yellow circle and the characteristic latitudinal offsets for the WIC (blue), SI-13 (red) and SI-12 (green) proxies are shown relative to the DMSP proxy. Latitude difference is shown by the radial co-ordinates.

\section{Discussion}

In this paper we have compared the latitude of the OCB as estimated from auroral images from the WIC, SI-12 and SI13 FUV detectors onboard the IMAGE satellite and with the latitude of the OCB as estimated by DMSP particle precipitation measurements.

Figures 3 and 6 show that at most MLTs the average modal difference in the latitude between the WIC, SI-12 and SI-13 OCB proxies is statistically small $\left(<1^{\circ}\right)$, although at any one 
MLT bin differences may be larger. Larger average discrepancies are seen in the evening and predawn sectors. In the evening (18:00-23:00 MLT) sector SI-12 OCB proxies can be located between 1-2 degrees equatorward of WIC and SI13 OCB proxies and 1-2 degrees poleward of WIC and SI-13 OCB proxies in the predawn (03:00-06:00 MLT) sector. Below we will discuss how the magnetospheric current system may explain these differences.

In the dawn sector ionosphere region-1 Field Aligned Currents (FACs) are directed downward into the ionosphere and vice-versa in the evening sector. Equatorward of region-1 currents are region-2 FACs directed oppositely to region-1 FACs (Iijima and Potemra, 1978). Upward FACs are associated with down-going electrons and are therefore associated with the WIC and SI-13 electron auroral emission (although these also have a small contribution from secondary electrons produced by the proton aurora). The dominant charge carrier associated with downward FACs is less clear but if they are assumed to be associated with down-going protons (CranMcGreehin and Wright, 2005) then we may expect downward FACs to be associated with the SI-12 auroral emission. We would therefore expect to see WIC and SI-13 auroral emission poleward of SI-12 emission in the evening sector and equatorward in the dawn sector, as seen in this study. It is also interesting to note that the offsets between FUV proxies is small on the dayside and do not follow the morphology of the FACs. However, it is known that the location at which region-1 and region-2 currents switch from upwards to downwards varies over a large MLT range in this sector depending on the orientation of the IMF $B_{y}$ component (Weimer, 2001). Over large time scales the average of the IMF $B_{y}$ component is close to zero and therefore the boundary offsets seen are also close to zero in this MLT sector.

Figures 5 and 6 show that in the predawn sector the SI-12 OCB proxy is in better agreement with DMSP OCB proxy than either the WIC or SI-13 OCB proxies, but is still located over $1^{\circ}$ equatorward of the DMSP proxy, which is thought to be the best proxy for the OCB. Previous studies (Wild et al., 2004) have suggested that WIC may be an unreliable proxy for the OCB in the predawn sector whilst others (Hubert et al., 2006) have used SI-12 in preference to SI-13 or WIC when determining the global OCB due to the reduced dayglow in SI-12 rather than the accuracy of the detector in determining the location of the OCB. Our study confirms that SI-12 may be a better estimation for the OCB in the predawn sector. However, Figs. 5 and 6 show that at all later magnetic local times the WIC and SI-13 OCB proxies are either in better agreement with DMSP proxies or as good as the SI12 proxy. The most accurate global determination of the OCB may therefore be obtained by using the individual FUV detector which has the best correlation with the DMSP OCB proxy in any one MLT sector. For example SI12 in the 01:00-07:00 MLT range, WIC at 07:00-08:00, SI13 at 08:00-16:00 MLT, SI12 at 16:00-17:00 MLT, SI13 at 17:00-22:00 and WIC at 22:00-01:00 MLT. For single in-

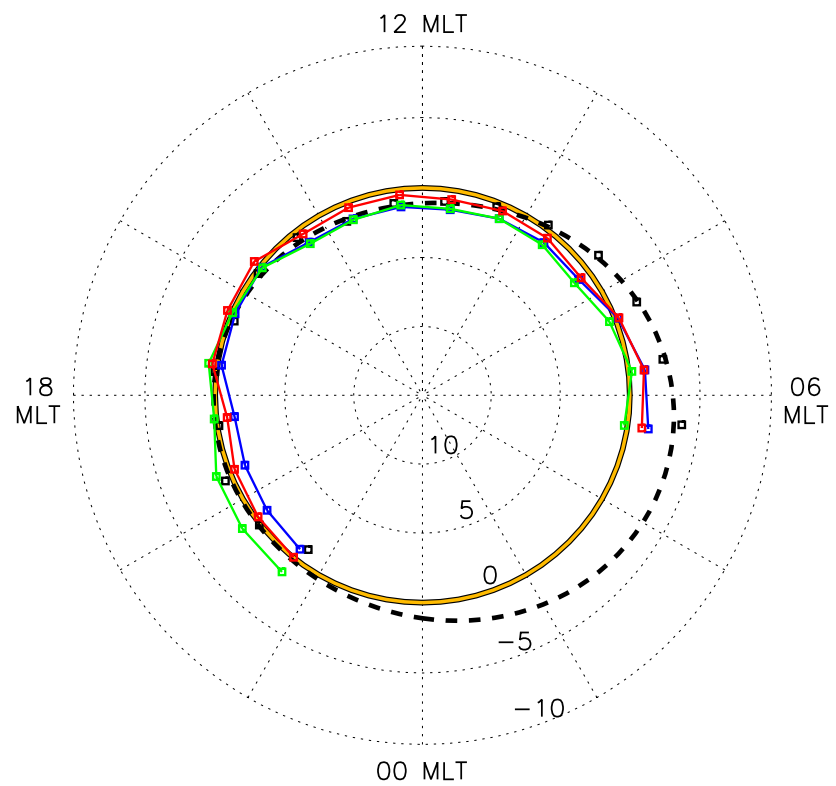

Fig. 6. Optimally estimated latitude differences between WIC (dark blue), SI-13 (red) and SI-12 (green) OCB proxies relative to the latitude of the DMSP OCB proxy (yellow). The fit of Carbary et al. (2003) for Polar UVI boundaries is shown by the black dashed line. Latitude differences between DMSP and Polar UVI OCB proxies from Carbary et al. (2003) are shown by black rectangles and the black dashed line shows the extrapolated fit to all MLTs. Radial co-ordinates represent latitude difference relative to the DMSP OCB proxy in degrees.

strument studies WIC may produce the best proxies for the global OCB due to its good global correlation with the DMSP OCB proxy and its better spatial resolution. We also note that the FUV OCB proxies are found poleward of the equivalent DMSP proxy at dayside MLTs. Near noon there is frequently more particle flux on open field lines (cusp, mantle, etc.) than closed, particularly in SI-12 which is likely seeing cusp protons. DMSP counts the cusp precipitation as open and so places the OCB equatorward of the FUV emission.

Figures 5 and 6 confirm that systematic differences exist between estimations of the OCB from DMSP particle precipitation measurements and FUV auroral imagers, similar to the differences seen between DMSP and radar derived OCB proxies as presented by Chisham et al. (2007). These systematic differences may be used to "correct" auroral boundaries to give a more accurate determination of the OCB. Unfortunately due to the low number of correlations found between the DMSP and FUV OCB proxies and the lack of data available in the predawn sector (where the offset is at its largest) the uncertainties involved in this correction are not insignificant. Although we have chosen to use the mode of the OCB proxy latitude differences and smoothed to give the most reliable distributions we have seen that using the mean of the latitude differences, or applying different degrees of smoothing, can change the fits to the DMSP-FUV OCB proxies 
differences in the predawn sector (where the fit is interpolated) by up to a degree. Therefore, we can only reliably trust the DMSP correction where DMSP data are available (06:00-21:00 MLT). However, as a best guess of the location of the OCB, applying this correction will statistically give a more accurate result than using the uncorrected FUV OCB proxies.

\section{Summary}

By comparing over $400000 \mathrm{OCB}$ proxies estimated from the IMAGE WIC, SI-13 and SI-12 FUV detectors and from auroral images obtained in December 2000 and December and January 2001-2002, we have shown that systematic differences exist between the latitude of the OCB as estimated by the three detectors. Statistically the OCB proxy differences are small $\left(<1^{\circ}\right)$ except in the predawn sector where the SI-12 OCB proxy is found, on average, to be located up to $2^{\circ}$ poleward of the WIC and SI-13 OCB proxies and in the evening sector where the SI-12 OCB proxy can be located up to $2^{\circ}$ equatorward of SI-13 and WIC OCB proxies.

Correlating FUV OCB proxies with the DMSP OCB proxy confirms that the SI-12 OCB proxy is found poleward of the WIC and SI-13 OCB proxies in the predawn sector, in better agreement with the DMSP OCB proxy. The WIC and SI-13 OCB proxies are found to be in better agreement with the DMSP OCB proxy at all other MLTs. A systematic difference between the DMSP and FUV OCB proxies is found and may be used to correct the FUV OCB proxies to give a more accurate global estimation of the OCB.

Acknowledgements. PDB was supported by a PPARC/STFC CASE award, grant no. PPA/S/C/2006/04488. The authors would like to thank the many dedicated scientists who have made the IMAGE mission a success. B. Hubert is supported by the Belgian National Fund for Scientific Research (FNRS).

Topical Editor I. A. Daglis thanks G. Blanchard and J. Wild for their help in evaluating this paper.

\section{References}

Baker, J. B., Clauer, C. R., Ridley, A. J., Papitashvili, V. O., Brittnacher, M. J., and Newell, P. T.: The nightside poleward boundary of the auroral oval as seen by DMSP and the Ultraviolet Imager, J. Geophys. Res., 105, 21 267-21 280, 2000.

Baker, K. B., Dudeney, J. R., Greenwald, R. A., Pinnock, M., Newell, P. T., Rodger, A. S., Mattin, N., and Meng, C. I.: Hf Radar Signatures of the Cusp and Low-Latitude BoundaryLayer, J. Geophys. Res., 100, 7671-7695, 1995.

Blanchard, G. T., Ellington, C. L., Lyons, L. R., and Rich, F. J.: Incoherent scatter radar identification of the dayside magnetic separatrix and measurement of magnetic reconnection, J. Geophys. Res., 106, 8185-8195, 2001.

Blanchard, G. T., Lyons, L. R., de la Beaujardiere, O., Doe, R. A., and Mendillo, M.: Measurement of the magnetotail reconnection rate, J. Geophys. Res., 101, 15 265-15 276, 1996.
Blanchard, G. T., Lyons, L. R., and Samson, J. C.: Accuracy of using 6300 angstrom auroral emission to identify the magnetic separatrix on the nightside of earth, J. Geophys. Res., 102, 96979703, 1997.

Blanchard, G. T., Lyons, L. R., Samson, J. C., and Rich, F. J.: Locating the Polar-Cap Boundary from Observations of 6300 Angstrom Auroral Emission, J. Geophys. Res., 100, 7855-7862, 1995.

Brittnacher, M., Fillingim, M., Parks, G., Germany, G., and Spann, J.: Polar cap area and boundary motion during substorms, J. Geophys. Res., 104, 12 251-12 262, 1999.

Carbary, J. F., Sotirelis, T., Newell, P. T., and Meng, C. I.: Auroral boundary correlations between UVI and DMSP, J. Geophys. Res., 108, 1018, doi:10.1029/2002JA009378, 2003.

Chisham, G., Freeman, M. P., Abel, G. A., Lam, M. M., Pinnock, M., Coleman, I. J., Milan, S. E., Lester, M., Bristow, W. A., Greenwald, R. A., Sofko, G. J., and Villain, J.-P.: Remote sensing of the spatial and temporal structure of magnetopause and magnetotail reconnection from the ionosphere, Rev. Geophys., 46, RG1004, doi:10.1029/2007RG000223, 2008.

Chisham, G. and Freeman, M. P.: A technique for accurately determining the cusp-region polar cap boundary using SuperDARN HF radar measurements, Ann. Geophys., 21, 983-996, 2003, http://www.ann-geophys.net/21/983/2003/.

Chisham, G., Freeman, M. P., Sotirelis, T., Greenwald, R. A., Lester, M., and Villain, J. P.: A statistical comparison of SuperDARN spectral width boundaries and DMSP particle precipitation boundaries in the morning sector ionosphere, Ann. Geophys., 23, 733-743, 2005, http://www.ann-geophys.net/23/733/2005/.

Chisham, G., Lester, M., Milan, S. E., Freeman, M. P., Bristow, W. A., Grocott, A., McWilliams, K. A., Ruohoniemi, J. M., Yeoman, T. K., Dyson, P. L., Greenwald, R. A., Kikuchi, T., Pinnock, M., Rash, J. P. S., Sato, N., Sofko, G. J., Villain, J. P., and Walker, A. D. M.: A decade of the Super Dual Auroral Radar Network (SuperDARN): scientific achievements, new techniques and future directions, Surv. Geophys., 28, 33-109, 2007.

Chisham, G., Pinnock, M., Coleman, I. J., Hairston, M. R., and Walker, A. D. M.: An unusual geometry of the ionospheric signature of the cusp: implications for magnetopause merging sites, Ann. Geophys., 20, 29-40, 2002, http://www.ann-geophys.net/20/29/2002/.

Chisham, G., Pinnock, M., and Rodger, A. S.: The response of the HF radar spectral width boundary to a switch in the IMF B-y direction: Ionospheric consequences of transient dayside reconnection?, J. Geophys. Res., 106, 191-202, 2001.

Cran-McGreehin, A. P. and Wright, A. N.: Electron acceleration in downward auroral field-aligned currents, J. Geophys. Res.-Space Phys., 110, A10S15, doi:10.1029/2004JA010898, 2005.

Elphinstone, R. D., Murphree, J. S., and Cogger, L. L.: What is a global auroral substorm?, Rev. Geophys., 34, 169-232, 1996.

Hubert, B., Milan, S. E., Grocott, A., Blockx, C., Cowley, S. W. H., and Gerard, J. C.: Dayside and nightside reconnection rates inferred from IMAGE FUV and Super Dual Auroral Radar Network data, J. Geophys. Res., 111, A03217, doi:10.1029/2005JA011140, 2006.

Iijima, T. and Potemra, T. A.: Large-Scale Characteristics of FieldAligned Currents Associated with Substorms, J. Geophys. Res.Space Phys., 83, 599-615, 1978. 
Kauristie, K., Weygand, J., Pulkkinen, T. I., Murphree, J. S., and Newell, P. T.: Size of the auroral oval: UV ovals and precipitation boundaries compared, J. Geophys. Res., 104, 2321-2331, 1999.

Lam, M. M., Pinnock, M., and Donovan, E. F.: Observations of nightside magnetic reconnection during substorm growth and expansion phases, J. Geophys. Res., 111, A05209, doi:10.1029/2005JA011356, 2006.

Lester, M., Milan, S. E., Besser, V., and Smith, R.: A case study of HF radar spectra and $630.0 \mathrm{~nm}$ auroral emission in the premidnight sector, Ann. Geophys., 19, 327-339, 2001, http://www.ann-geophys.net/19/327/2001/.

Mende, S. B., Frey, H. U., Morsony, B. J., and Immel, T. J.: Statistical behavior of proton and electron auroras during substorms, J. Geophys. Res.-Space Phys., 108, 1339, doi:10.1029/2002JA009751, 2003.

Mende, S. B., Heetderks, H., Frey, H. U., Lampton, M., Geller, S. P., Abiad, R., Siegmund, O. H. W., Tremsin, A. S., Spann, J., Dougani, H., Fuselier, S. A., Magoncelli, A. L., Bumala, M. B., Murphree, S., and Trondsen, T.: Far ultraviolet imaging from the IMAGE spacecraft. 2. Wideband FUV imaging, Space Sci. Rev., 91, 271-285, 2000a.

Mende, S. B., Heetderks, H., Frey, H. U., Stock, J. M., Lampton, M., Geller, S. P., Abiad, R., Siegmund, O. H. W., Habraken, S., Renotte, E., Jamar, C., Rochus, P., Gerard, J. C., Sigler, R., and Lauche, H.: Far ultraviolet imaging from the IMAGE spacecraft. 3. Spectral imaging of Lyman-alpha and OI $135.6 \mathrm{~nm}$, Space Sci. Rev., 91, 287-318, 2000b.

Milan, S. E., Lester, M., Cowley, S. W. H., Moen, J., Sandholt, P. E., and Owen, C. J.: Meridian-scanning photometer, coherent HF radar, and magnetometer observations of the cusp: a case study, Ann. Geophys., 17, 159-172, 1999, http://www.ann-geophys.net/17/159/1999/.
Milan, S. E., Lester, M., Cowley, S. W. H., Oksavik, K., Brittnacher, M., Greenwald, R. A., Sofko, G., and Villain, J. P.: Variations in the polar cap area during two substorm cycles, Ann. Geophys., 21, 1121-1140, 2003, http://www.ann-geophys.net/21/1121/2003/.

Newell, P. T., Burke, W. J., Sanchez, E. R., Meng, C. I., Greenspan, M. E., and Clauer, C. R.: The Low-Latitude Boundary-Layer and the Boundary Plasma Sheet at Low Altitude - Prenoon Precipitation Regions and Convection Reversal Boundaries, J. Geophys. Res., 96, 21 013-21 023, 1991.

Sandholt, P. E., Farrugia, C. J., Oieroset, M., Stauning, P., and Denig, W. F.: Auroral activity associated with unsteady magnetospheric erosion: Observations on December 18, 1990, J. Geophys. Res., 103, 2309-2317, 1998.

Sotirelis, T. and Newell, P. T.: Boundary-oriented electron precipitation model, J. Geophys. Res., 105, 18 655-18 673, 2000.

Weimer, D. R.: Maps of ionospheric field-aligned currents as a function of the interplanetary magnetic field derived from Dynamics Explorer 2 data, J. Geophys. Res.-Space Phys., 106, 12 889-12 902, 2001.

Wild, J. A., Milan, S. E., Owen, C. J., Bosqued, J. M., Lester, M., Wright, D. M., Frey, H., Carlson, C. W., Fazakerley, A. N., and Reme, H.: The location of the open-closed magnetic field line boundary in the dawn sector auroral ionosphere, Ann. Geophys., 22, 3625-3639, 2004, http://www.ann-geophys.net/22/3625/2004/. 